Artículo de revisión y/o actualización de literatura

\title{
Eficacia de los programas de musicoterapia: un metanálisis cualitativo
}

Eficácia dos programas de musicoterapia: uma meta-análise qualitativa Efficacy of music therapy programs: a qualitative meta-analysis

\author{
María José González-Ojea ${ }^{a}$ (D), Sara Domínguez-Lloriab (D), Margarita Pino-Juste ${ }^{a}$ (D) \\ ${ }^{a}$ Universidad de de Vigo - UVIGO, Vigo, España. \\ bUniversidad Santiago de Compostela - USC, Santiago de Compostela, España.
}

Cómo citar: González-Ojea, M. J., Domínguez-Lloria, S., \& Pino-Juste, M. (2021). Eficacia de los programas de musicoterapia: un metanálisis cualitativo. Cadernos Brasileiros de Terapia Ocupacional, 29, e2976. https://doi.org/10.1590/2526-8910.ctoAR2255

\begin{abstract}
$\underline{\text { Resumen }}$
El aumento de enfermedades transmisibles justifica la necesidad de verificar la eficacia no farmacológica de los tratamientos alternativos. La música promueve las interacciones sociales y el trabajo en grupo, ayuda en varios aspectos del aprendizaje humano, la emoción y la memoria, y puede convertirse en una herramienta terapéutica complementaria. El objetivo de este estudio fue analizar la evidencia de la eficacia de la musicoterapia como tratamiento no farmacológico mediante un diseńo descriptivo retrospectivo utilizando como parámetros de búsqueda: "musicoterapia" o "programas musicales" y "salud" en las bases de datos Scopus y Web of Science. Entre los principales resultados, destacamos que los programas de intervención que utilizan la música como tratamiento coadyuvante no farmacológico en enfermedades no transmisibles ofrecen resultados positivos y significativos sobre la mejora de las patologías en las que interviene. A partir de estos resultados, sería importante ampliar la formación de los profesionales de la salud en la aplicación de técnicas basadas en el arte y específicamente en la musicoterapia.
\end{abstract}

Palabras-clave: Música, Salud, Terapias Complementarias.

\section{Resumo}

O aumento das doenças transmissíveis justifica a necessidade de verificar a eficácia não farmacológica de tratamentos alternativos. A música promove interaçóes sociais e trabalho em grupo, auxilia em vários aspectos da aprendizagem humana, emoção e memória, e pode se tornar uma ferramenta terapêutica complementar. O objetivo deste estudo foi analisar a eficácia da musicoterapia como tratamento não farmacológico por meio de um desenho descritivo retrospectivo utilizando como parâmetros de busca: "musicoterapia" ou "programas de música" e "saúde" nas bases de dados Scopus e Web of Science. Dentre os principais resultados, destacamos que os programas de intervenção que utilizam a música como coadjuvante não 
farmacológico nas doenças não transmissíveis oferecem resultados positivos e significativos na melhora das patologias em que intervém. Com base nesses resultados, seria importante ampliar a formação dos profissionais de saúde na aplicação de técnicas baseadas na arte e especificamente na musicoterapia.

Palavras-chave: Música, Saúde, Terapias Complementares.

\begin{abstract}
$\underline{\text { Abstract }}$
The increase in communicable diseases justifies the need to verify the nonpharmacological efficacy of alternative treatments. Music promotes social interactions and group work, helps in various aspects of human learning, emotion, and memory, and can become a complementary therapeutic tool. This study aimed to analyse the efficacy of music therapy as a non-pharmacological treatment through a retrospective descriptive design using as search parameters: "music therapy" or "music programs" and "health" in the Scopus and Web of Science databases. Among the main results, we highlight that the intervention programs that use music as an adjunctive nonpharmacological treatment in non-communicable diseases offer positive and significant results on the improvement of the pathologies in which it intervenes. Based on these results, it would be important to expand the training of health professionals in the application of techniques based on art and specifically in music therapy.
\end{abstract}

Keywords: Music, Health, Complementary Therapies.

\title{
Introducción
}

La carga de mortalidad atribuida a las Enfermedades no transmisibles (ENT) sigue en aumento a pesar de las políticas sanitarias que se han puesto en marcha en las últimas décadas basadas en una buena alimentación, la práctica regular de actividad física y evitar hábitos nocivos como beber alcohol, fumar o ingerir sustancias tóxicas.

Según la World Health Organization (2018) las ENT matan en el mundo a 41 millones de personas cada ańo, lo que equivale al $71 \%$ de todas las muertes a nivel mundial. Además, cada ańo, 15 millones de personas mueren a causa de una de las citadas ENT entre las edades de 30 y 69 ańos. Las enfermedades cardiovasculares representan la mayoría de las muertes por ENT, 17,9 millones de personas al ańo, seguidas del cáncer (9,0 millones), las enfermedades respiratorias (3,9 millones) y la diabetes (1,6 millones). Estos 4 grupos de enfermedades representan más del $80 \%$ de todas las muertes prematuras por ENT. Por tanto, la prevención, la detección temprana y el tratamiento de estas enfermedades se convierten en objetivos clave para el sistema sanitario de todos los países.

El cerebro humano y la música están estrechamente relacionados. La música tiene el poder demostrado de hacer cambios en el cerebro sano, en su estructura y función, mejorando sentimientos negativos (Tian et al., 2020; Penhune, 2020). También influye en muchas características del comportamiento de las personas, promueve las interacciones sociales y el trabajo en grupo, ayuda en varios aspectos del aprendizaje y la memoria del ser humano, aporta motivación y es de naturaleza gratificante, ayudando a nivel emocional (Barbeau \& Cossette, 2019; Harvey, 2020; Koelsch, 2020; Ye, 2020). Hace más fácil 
unirse y trabajar en grupo, ayuda en la cohesión social. Podemos utilizarla de manera terapéutica, pero también bélicamente cambiando la intensidad del sonido (JausetBerrocal, 2018).

Sin embargo, aunque en el ámbito médico no hay gran interés por su utilización y falta mucha evidencia científica, la música ha sido reconocida como un método no farmacológico para ayudar a reducir el dolor en los procedimientos médicos (Pedersen et al., 2020; Gogoularadja \& Bakshi, 2020), como por ejemplo en las cesáreas, donde el uso combinado de la analgesia y la música tiene mayor efectividad en el control del dolor que la analgesia por sí sola (Zimpel et al., 2020), para estabilizar la frecuencia respiratoria y la tensión arterial en pacientes intervenidos quirúrgicamente (Ecer \& Saritaş, 2019), o para mejorar la respiración y la resistencia física tras la participación en bandas musicales (Barbeau \& Cossette, 2019).

En este análisis se entiende la musicoterapia como:

[...] el uso profesional de la música y sus elementos como una intervención en entornos médicos, educacionales y cotidianos con individuos, grupos, familias o comunidades que buscan optimizar su calidad de vida y mejorar su salud y bienestar físico, social, comunicativo, emocional, intelectual y espiritual. La investigación, la práctica, la educación y el entrenamiento clínico en musicoterapia están basados en estándares profesionales acordes a contextos culturales, sociales y políticos (Haase, 2012, p. 192).

La evidencia científica sobre la eficacia del arte en el tratamiento de diferentes patologías se ha puesto de manifiesto en el estudio de Fancourt \& Finn (2019) donde se señalan los efectos positivos de las artes en la salud y el bienestar, concretamente en la prevención de enfermedades, la promoción de la salud y el manejo y tratamiento de enfermedades a lo largo de la vida. En el análisis sobre qué tipo de interacciones artísticas se consideran arte, aparecen las artes escénicas en donde se incluyen la música, danza, teatro, canto y el cine (Davies et al., 2012). Es decir, la música no es sólo una fuente de entretenimiento y conocimiento, sino que puede convertirse en una herramienta terapéutica complementaria cuando aparecen una serie de condiciones médicas donde existen limitaciones a la terapia actual o como tratamiento coadyuvante (Miranda et al., 2017).

Dado el aumento de las ENT y las ventajas que puede tener la musicoterapia para la salud, en este estudio se pretende analizar la evidencia científica existente sobre la eficacia de la musicoterapia como tratamiento no farmacológico teniendo en cuenta la franja etaria de los pacientes y las patologías y los síntomas tratados.

\section{Método}

Se ha realizado un estudio descriptivo retrospectivo recopilando la información más relevante sobre la utilización de la música como tratamiento no farmacológico (Day, 2005). Para la localización de los documentos bibliográficos se utilizaron varias fuentes documentales. En primer lugar, se procedió a realizar una búsqueda en las siguientes bases de datos: Scopus y Web of Science. Además, los artículos de revisión y las revisiones sistemáticas relacionadas sobre esta temática se utilizaron como guías para obtener otros estudios que se ajustan a los criterios de inclusión. 
Las palabras clave utilizadas, para llevar a cabo la búsqueda de información, fueron: "music therapy", "health" y "music programs". El parámetro de búsqueda fue: "music therapy" o "music programs" y "health" en el título, resumen o palabras clave. Se utilizó el mismo logaritmo de búsqueda en ambas bases de datos citadas anteriormente. Se utilizaron términos en inglés ya que las bases de datos utilizadas indizan los artículos en este idioma.

\section{Criterios de inclusión y exclusión}

Una vez revisados los resultados obtenidos, se llevó a cabo la selección final de los estudios a analizar. En la búsqueda y selección de artículos se establecieron los siguientes criterios de inclusión: estudios del 2015 al 2020 y publicados en inglés, castellano o portugués. Además, se ha tenido en cuenta como indicador de calidad que los estudios estuviesen sujetos a un proceso de revisión por pares antes de su publicación y debían poseer una metodología coherente y clara.

De los 167 inicialmente encontrados se excluyeron 117 ya que no se relacionaban con los efectos de la musicoterapia o sobre programas de intervención donde la música era la principal técnica. Por tanto, no se incluyeron estudios sobre danza aeróbica al considerarla una práctica deportiva. Se excluyeron también aquellos estudios donde los participantes tuviesen conocimientos musicales o fuesen músicos profesionales, dado que sus conocimientos musicales podrían afectar a la eficacia del programa.

Por tanto, siguiendo a Page et al. (2021) se ha diseñado el siguiente Esquema 1 en PRISMA:

Identificación de estudios a través de bases de datos y registros

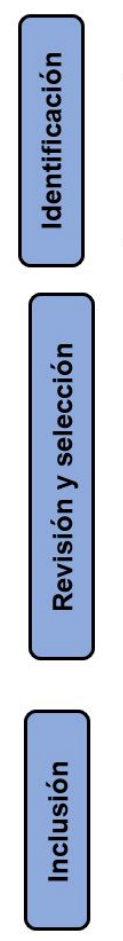

Estudios identificados a través de la búsqueda en base de datos: $(n=167)$

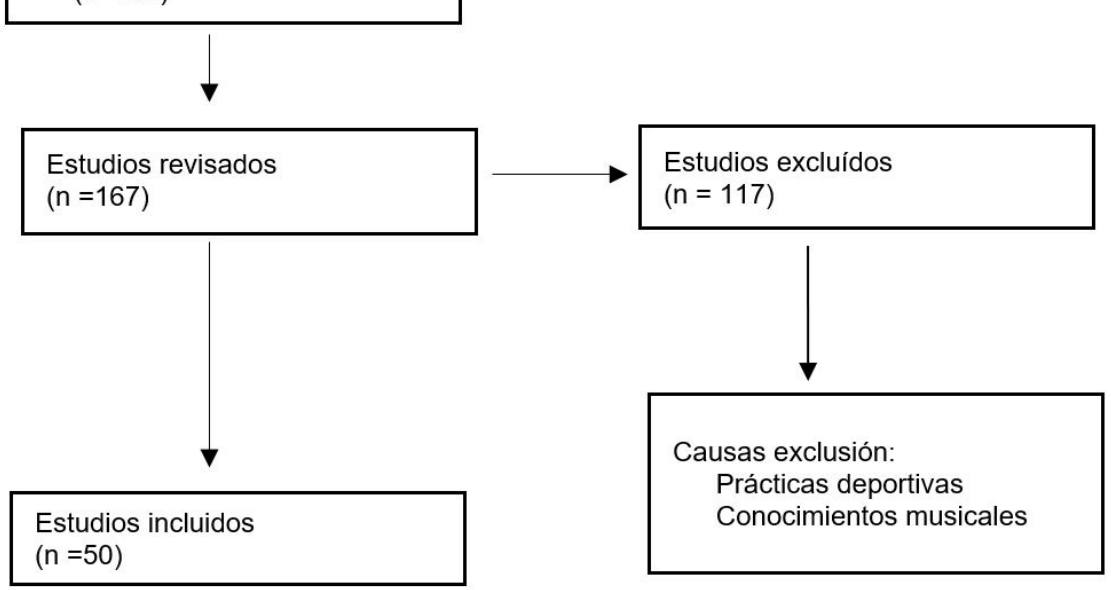

Figura 1. Elaboración propia. 


\section{Resultados}

Para organizar los resultados se han diseñado una serie de categorías que facilitan su comprensión e interpretación.

\section{Métodos de investigación utilizados para el diseño y evaluación de programas}

Para valorar la eficacia de los programas, como se muestra en la Figura 2, se ha utilizado una metodología cuantitativa en 26 estudios (52\%). En 24 de éstos (92\%) los instrumentos seleccionados han sido los cuestionarios y las escalas de medición mediante un diseño pretest-postest. Únicamente en un estudio se mide la satisfacción de los pacientes después de la realización de una sesión de musicoterapia (Mandel et al., 2019) siendo el único estudio que no realiza pretest.

Otros 16 programas presentan una metodología de investigación cualitativa (32\%). 6 de ellas son revisiones teóricas (37.5\%) (Loewy, 2020; Philip et al., 2019; Sheppard \& Broughton, 2020; Vinciguerra et al., 2019; Wei et al., 2020; Gramaglia et al., 2019) o metaanálisis en 4 programas (25\%) (Koehler et al., 2020; Lin et al., 2020; Wang et al., 2020; Lopes-Junior et al., 2016). La media de estudios analizados en cada revisión es 15.

Algunos estudios utilizan diferentes técnicas cualitativas combinando el registro observacional y los grupos de discusión (Hall et al., 2019), el registro observacional y entrevista (Millar et al., 2020), la entrevista y el grupo de discusión (Perkins et al., 2016), e incluso triangulan las técnicas utilizando la entrevista, el registro observacional y diarios de práctica de los participantes (MacRitchie et al., 2020).

El estudio Ford et al. (2018) emplea la indagación colaborativa sustentada en el enfoque constructivista para evaluar el programa. Y algunos estudios emplean un único instrumento cualitativo, bien las entrevistas (Rosado, 2019; Windle et al., 2020) o bien el registro observacional (Sharififard et al., 2020).

Por último, 8 programas se evalúan mediante una metodología mixta (16\%). La mayoría de ellos, 6 programas $(75 \%)$ presentan un método cuantitativo a través de cuestionario con un diseño pretest-postest y como técnica cualitativa la entrevista (Calo et al., 2020; Gulliver et al., 2019; Hirschberg et al., 2020; Low et al., 2020; Riggins \& McLennon, 2020; Uhlig et al., 2016).

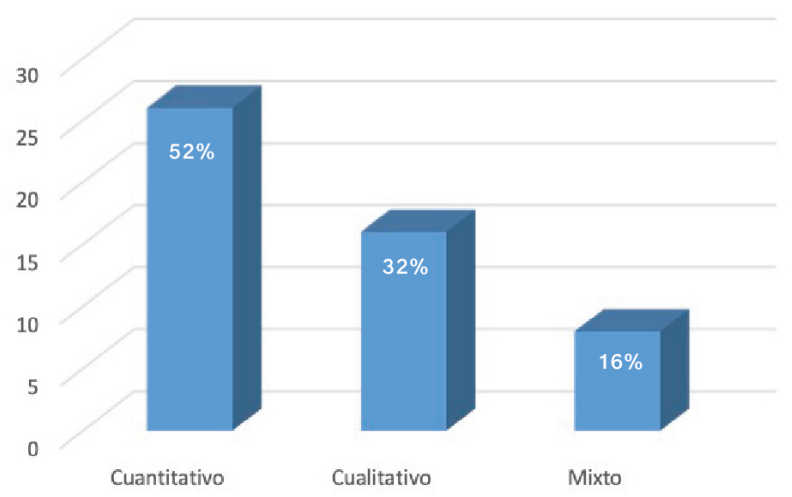

Figura 2. Tipo de método utilizado para valorar la eficacia del programa. 
Los métodos de investigación empleados para cada franja etaria son en el caso del programa destinado a niños la metodología mixta utilizando como instrumento cualitativo el registro observacional. En el caso de los jóvenes 4 de los programas (25\%) emplean metodología cuantitativa, 2 metodología mixta (25\%) y 2 metodología cualitativa (25\%).

La mayoría de los programas (6) destinados a adultos presentan una metodología cuantitativa (54.54\%), 3 metodología cualitativa (27.27\%) y 2 metodología mixta (18.18\%).

Los programas destinados a un rango de varias edades emplean el método cuantitativo en 9 de los programas (el 50\%), método cualitativo en 8 programas (el 44.4\%) y solamente en $1(5.55 \%)$ metodología mixta.

En la franja etaria de la tercera edad el método cuantitativo se emplea en 7 de ellos programas (58.33\%), el cualitativo en 3 (25\%) y el mixto en otros 2 programas (el 16.66\%).

El ámbito de implementación de los programas se llevo a cabo en su mayoría en hospitales (40\%), en pacientes no hospitalizados el $24 \%$, en residencias de ancianos el $20 \%$, el $8 \%$ en hospitales de salud mental, dos programas se llevaron a cabo en una asociación, 1 programa en una comunidad marginal y otro en una iglesia.

Sobre las patologías y síntomas asociados tratados en los programas hemos realizado una agrupación para facilitar la interpretación de los datos.

\section{Patologías en función de las franjas etarias}

Con respecto a las franjas etarias (Figura 3) con las que se implementan los programas nos encontramos que casi no existen programas centrados en la niñez. Solamente un programa $(2 \%)$ está destinado a niños. En la franja de los jóvenes se han analizado ocho programas (16\%), para adultos $11(22 \%), 12$ en las personas mayores (24\%). La mayoría de los programas (18) basados en la música como técnica terapéutica se realizan en varias franjas de edad de forma conjunta (36\%).

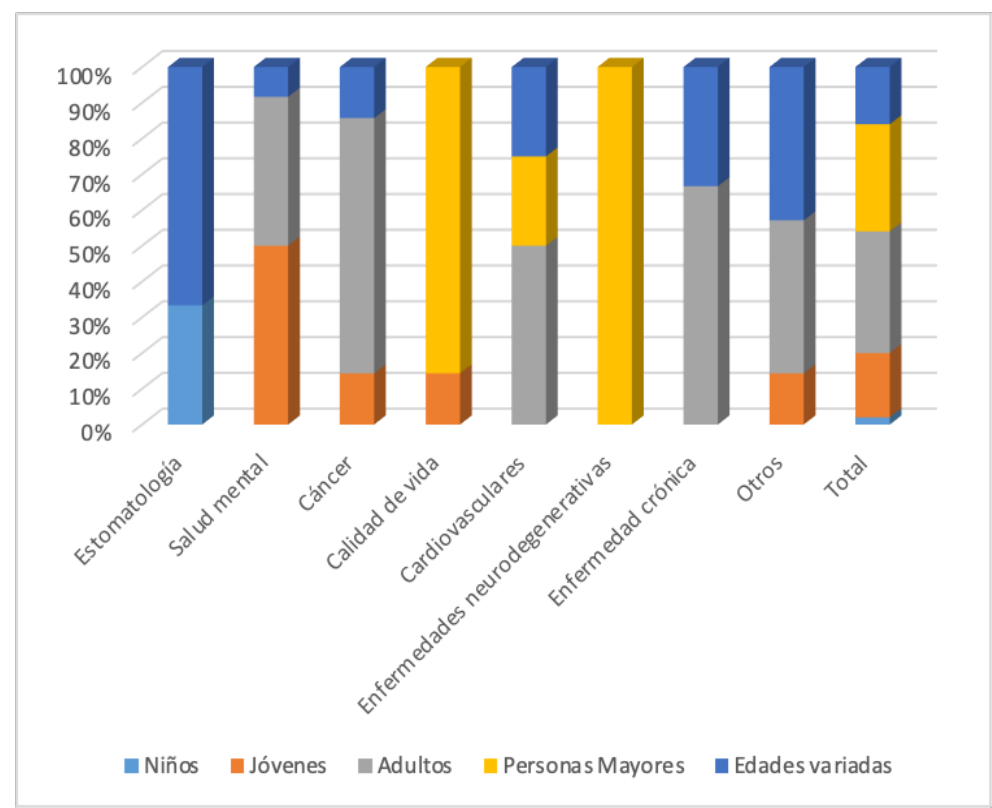

Figura 3. Porcentaje de las diferentes patologias tratadas en los programas de musicoterapia en las diferentes franjas etarias. 
Si nos centramos en las patologías más encontradas en los estudios analizados podríamos clasificarlas en las siguientes categorías, reflejadas en la Figura 4.

\section{Bienestar y calidad de vida}

La mayoría de los programas (10) tratan condiciones referidas al bienestar y la calidad de vida (20\%). Los indicadores tratados son la confianza y el compromiso (Calo et al., 2020; Millar et al., 2020), las habilidades sociales (Ford et al., 2018; MacRitchie et al., 2020; Millar et al., 2020), la calidad de vida (Fujita et al., 2016; Giuli et al., 2020; Sheppard \& Broughton, 2020; Uhlig et al., 2016) y el interés por la vida (Johnson et al., 2020; Riggins \& McLennon, 2020).

La práctica de la música mejora los factores cognitivos en personas de la tercera edad y, el ambiente que se crea al realizar estas prácticas mejora considerablemente el bienestar físico (MacRitchie et al., 2020; Ford et al., 2018; Fujita et al., 2016; Riggins \& McLennon, 2020). Algunos estudios constatan que esta mejora de la calidad de vida se da también en colectivos y poblaciones grandes y diversas (Sheppard \& Broughton, 2020), sin embargo, es importante que se mantengan estas prácticas en el tiempo ya que su naturaleza transitoria y a corto plazo puede resultar problemática en grupos de difícil acceso (Millar et al., 2020).

También se ha comprobado la eficacia y el impacto positivo de la musicoterapia sobre la ansiedad en pacientes que padecían patologías cardiovasculares (Ashok et al., 2019).

\section{Salud mental}

El número de programas que tratan la salud mental a través de la musicoterapia son $\operatorname{diez}(20 \%)$. Los síntomas que trabajan son la ansiedad (Archambault et al., 2019; Ashok et al., 2019; Travis Junior et al., 2019), la calidad de vida (Hall et al., 2019), la depresión (Kayak et al., 2016; Perkins et al., 2016; Rosado, 2019; Travis Junior et al., 2019), y el bienestar (Kwok, 2019; Windle et al., 2020). En la recuperación de los jóvenes, la intervención a través de la musicoterapia proporciona un medio eficaz y adaptativo para la regulación de los estados de ánimo (Archambault et al., 2019; Rosado, 2019). Autores como Hall et al. (2019) afirman que existe una mejoría de la inclusión social a través de la música en pacientes con enfermedades mentales.

\section{Cáncer}

Existen siete programas de musicoterapia (14\%) que se implementan con enfermos de cáncer. Los beneficios de estos programas son la reducción de los síntomas de la ansiedad y la depresión (Gramaglia et al., 2019; Koehler et al., 2020; Lee et al., 2020; López et al., 2019), la mejora de la calidad de vida y el bienestar (Koehler et al., 2020; Lee et al., 2020; Valero-Cantero et al., 2020; Wei et al., 2020) y, la reducción del dolor y el estrés (Koehler et al., 2020; Lee et al., 2020; Lopes-Junior et al., 2016). La participación en programas de musicoterapia tanto para los enfermos de cáncer como para sus cuidadores disminuyen la ansiedad y la tensión (Valero-Cantero et al., 2020). En investigaciones a través de ensayos aleatorios con pacientes oncológicos se determinó que la intervención a través de la musicoterapia contribuye a la mejora significativa de la angustia global, física y psicosocial que padecían, así como la sensación de bienestar (López et al., 2019; 
Gramaglia et al., 2019; Lee et al., 2020; Wei et al., 2020; Koehler et al., 2020; LopesJunior et al., 2016).

\section{Enfermedades neurodegenerativas}

La demencia es tratada en 6 de los programas (12\%), los síntomas tratados en este caso son la disminución de la función cognitiva (Castro et al., 2020; Domínguez-Chávez et al., 2019; Skingley et al., 2020; Wang et al., 2020), la depresión (Castro et al., 2020; Gulliver et al., 2019; Wang et al., 2020) y las habilidades sociales (Gold et al., 2019; Skingley et al., 2020). Skingley et al. (2020) y Gulliver et al. (2019) y Skingley et al. (2020) hablan de los efectos positivos de la musicoterapia con respecto al bienestar para personas con demencia y enfermedad de Alzheimer, insistiendo en la necesidad de implementarlos de forma continuada en el tiempo. Otros autores afirman que es necesario establecer protocolos estandarizados en función del estadio de la demencia (Wang et al., 2020). Giuli et al. (2020) y Domínguez-Chávez et al. (2019) han realizado proyectos que proporcionan pistas sobre el manejo de personas de la tercera edad afectadas por deterioro cognitivo seńalando que la música puede ayudar a prevenir la demencia. El uso de musicoterapia en personas con demencia ha dado resultados positivos con respecto a la reducción de la ansiedad y la depresión (Gold et al., 2019; Castro et al., 2020).

\section{Estrés}

Esta patología se trata en 4 programas (8\%). La sintomatología tratada es el trastorno de estrés postraumático y síntomas depresivos (Hirschberg et al., 2020); la ansiedad (Kishida et al., 2019) y el dolor (Lin et al., 2020; Low et al., 2020; Mandel et al., 2019). En el análisis se han encontrado mejoras en los pacientes a través de la creación de composiciones y creaciones propias reduciendo el estrés, la depresión y la ansiedad (Hirschberg et al., 2020; Windle et al., 2020).

\section{Patologías crónicas}

Las patologías crónicas engloban 7 estudios (14\%) y los síntomas tratados son diversos, en el caso de las dolencias cardiovasculares la sintomatología tratada es la ansiedad (Bittman et al., 2020; Shankar et al., 2020). En las patologías neurológicas se trabaja fundamentalmente en los síntomas referidos a la mejora de las habilidades sociales y comunicativas (Tillmann et al., 2020; Vinciguerra et al., 2019).

En el caso de patologías derivadas del dolor referido a la canulación para hemodiálisis los pacientes sometidos a audición musical terapéutica referían una disminución del dolor y la ansiedad durante el proceso (Kishida et al., 2019) provocando este mismo efecto ansiolítico en personas que presentaban trastorno del sueño (Loewy, 2020). En el caso de pacientes ortopédicos existen evidencias de que la música puede aliviar el dolor en el caso de procesos postoperatorios tanto si es usada como medicina musical o como musicoterapia (Lin et al., 2020). Estudios que revisan el uso de la música y la danza en el tratamiento de la enfermedad pulmonar crónica determinan que, aunque todavía la evidencia es limitada, existen beneficios biopsicosociales (Philip et al., 2019).

La fibromialgia es tratada en un único programa, mejorando la sintomatología de depresión y malestar (Espí-López et al., 2016). En otro programa en pacientes con 
dolencias pulmonares, la musicoterapia resulta ser efectiva para la mejora de la calidad de vida (Philip et al., 2019).

\section{Patologías estomatológicas}

En patologías estomatológicas se han detectado efectos positivos a la hora de aplicar la musicoterapia antes de cirugía periodontal funcionando como un ansiolítico no farmacológico efectivo sobre los signos vitales y la apertura de la boca (Kocaman \& Benli, 2019; Packyanathan et al., 2019). En algunos casos la musicoterapia combinada con otros métodos puede motivar a los nińos con discapacidad visual a mejorar su higiene bucodental (Sharififard et al., 2020). El total de programas asciende a $3(6 \%)$.

\section{Otras patologías}

La musicoterapia ha resultado ser efectiva también para patologías como la agenesia del cuerpo calloso donde un paciente adolescente sometido a un programa de percusión terapéutica evidenció mejoras en las habilidades motoras, comunicativas y en el comportamiento (Spak \& Card, 2019). Autores como Clayton et al. (2019) afirman que existen resultados prometedores en cuanto a la mejora de pacientes con accidente cerebrovascular agudo a través de la rehabilitación física y de la musicoterapia en la interacción social, las habilidades de comunicación, la función de las manos y la reducción de la frecuencia de las convulsiones (Clayton et al., 2019).

En el caso del síndrome de Rett se analizó como a través de la musicoterapia los pacientes mejoraron la habilidad comunicativa, la interacción social, el contacto visual, las convulsiones y la función de las manos mientras que reducía el estrés y el riesgo de depresión en sus cuidadores (Chou et al., 2019).

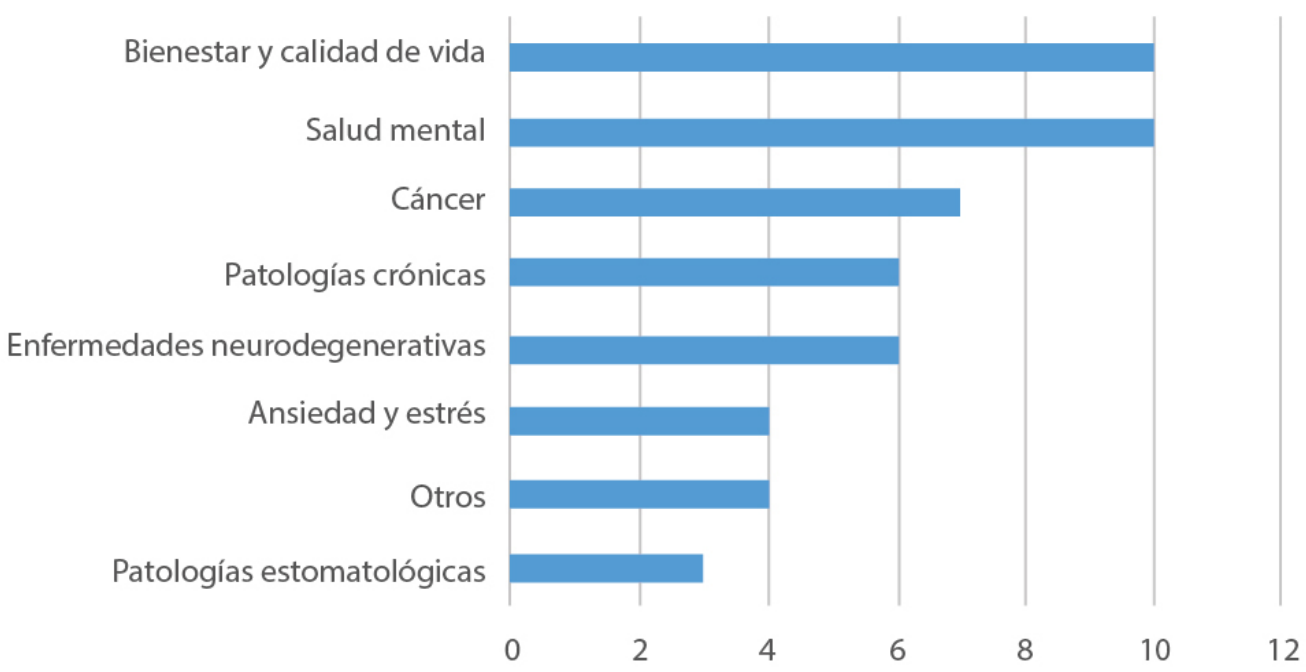

Figura 4. Patologías más habituales tratadas en los programas. 


\section{Discusión y Conclusiones}

Las actividades artísticas pueden considerarse intervenciones complejas o multimodales ya que combinan múltiples componentes que favorecen la promoción de la salud (Craig, et al., 2008). Las actividades artísticas pueden implicar compromiso estético, participación de la imaginación, activación sensorial, evocación de emociones y estimulación cognitiva. Y, dependiendo de su naturaleza, una actividad artística también puede implicar interacción social, actividad física o concentración, entre otras (Fancourt, 2017).

Las ventajas que produce la música se pueden aprovechar en determinadas terapias como tratamiento coadyuvante. La mayoría de los programas de musicoterapia se dirigen a personas adultas y tercera edad fundamentalmente ligados a salud mental (Archambault et al., 2019; Ashok et al., 2019; Travis Junior et al., 2019; Hall et al., 2019; Kayak et al., 2016; Perkins et al., 2016; Rosado, 2019; Kwok, 2019; Windle et al., 2020), y calidad de vida en enfermedades crónicas (Calo et al., 2020; Millar et al., 2020; Ford et al., 2018; MacRitchie et al., 2020; Fujita et al., 2016; Giuli et al., 2020; Sheppard \& Broughton, 2020; Uhlig et al., 2016; Johnson et al., 2020; Riggins \& McLennon, 2020). y degenerativas (Castro et al., 2020; Domínguez-Chávez et al., 2019; Skingley et al., 2020; Wang et al., 2020; Gulliver et al., 2019).

Para valorar de la eficacia de los programas implementados se han utilizado fundamentalmente métodos cuantitativos con instrumentos como el cuestionario y la escala, aunque para dar explicaciones a los resultados encontrados en un porcentaje importante de programas se han ayudado de instrumentos como los registros observacionales, las entrevistas o los diarios de campo. Cuando se utilizan solamente los métodos cualitativos los instrumentos de recogida de datos son las entrevistas, los grupos de discusión y los registros observacionales preferentemente.

Los diseños mixtos permiten la combinación de paradigmas que posibilitan el acercamiento a importantes problemas de investigación. La investigación mixta se fortalece con la incorporación de datos como imágenes, narraciones o verbalizaciones de las personas implicadas que dan sin duda un mayor sentido a los datos numéricos (Pérez, 2011; Alvariñas \& Pino-Juste, 2019).

Otra de las cuestiones que dan peso a este tipo de estudios es el uso de la triangulación, que funciona como un elemento verificador que ayuda al acercamiento del conocimiento del objeto del estudio (Moscoloni, 2005).

La triangulación permite visualizar un problema desde diferentes perspectivas de análisis y, por tanto, aumentar la consistencia y rigor de los resultados alcanzados. Esta técnica implica la combinación de varios enfoques metodológicos, de fuentes de datos, de teorías o de investigadores para analizar el mismo fenómeno. Permite conocerlo con una mayor profundidad y eliminar las debilidades o sesgos que aparecerían si solo se utilizara una técnica (Hernández, 2003).

Hemos podido comprobar que la mayoría (92\%) de los programas de intervención que utilizan la música como tratamiento no farmacológico ofrecen resultados positivos y significativos sobre la mejora de las patologías en las que interviene. Este efecto positivo es mucho más evidente en parámetros psicológicos tales como la ansiedad, la calidad de vida y el bienestar, además de mejorar las habilidades sociales y comunicativas. Únicamente encontramos dos estudios (4\%) que afirman que las intervenciones a través de la 
musicoterapia pueden contribuir a la mejora de los pacientes sin afirmarlo rotundamente (Fujita et al., 2016; Giuli et al., 2020) y dos estudios (4\%) resultan no concluyentes, ambos por ser un estudio piloto (Gold et al., 2019; Valero-Cantero et al., 2020).

Por tanto, la participación a través de programas de intervención que emplean la música reduce síntomas de ansiedad y depresión, así como mejora las capacidades sociales y emocionales (Travis Junior et al., 2019). También se constata que la musicoterapia reduce los niveles de ansiedad en pacientes con esquizofrenia crónica y puede utilizarse como terapia complementaria al tratamiento médico (Kayak et al., 2016). Autores como Perkins et al. (2016) concluyen que el uso de la creatividad y libertad musical son un factor clave en la reducción de la ansiedad, así como facilita las interacciones sociales. El uso de la musicoterapia para la reducción del estrés funciona como un reductor de ansiedad en los pacientes y sus cuidadores (Mandel et al., 2019). En el caso de los adolescentes la musicoterapia es eficaz para aliviar la psicopatología y reducir la ansiedad en los adolescentes (Sylvia \& Kwok, 2018).

La producción científica analizada permite concluir que la musicoterapia es un tratamiento no farmacológico coadyuvante en las enfermedades no transmisivas como el cáncer, las enfermedades crónicas y degenerativas o la salud mental para la mejora de la calidad de vida y la sintomatología ligada fundamentalmente al bienestar y a variables psicológicas como el estrés, la ansiedad o la autoestima. Y los programas diseñados tienen como población diana a adultos y personas mayores.

\section{Implicaciones Prácticas}

Queda patente los efectos beneficiosos de la musicoterapia como tratamiento no farmacológico coadyuvante en patologías con síntomas ligados a variables psicológicas (estrés, ansiedad, tensión), pero también al dolor ya que la utilización de sonidos, ritmos, armonías o melodías promueve la comunicación, las relaciones sociales, el movimiento o la expresión de emociones y sentimientos. En función de estos resultados sería importante ampliar la formación de los profesionales de la salud en la aplicación de técnicas basadas en el arte y diseñar programas en el ámbito hospitalario para niños y jóvenes, así como incorporar la musicoterapia como tratamiento no farmacológico de forma continuada en los centros hospitalarios, centros geriátricos $\mathrm{u}$ otras instituciones que tengan entre sus usuarios población con alguna patología donde se haya demostrado la eficacia de la musicoterapia como tratamiento coadyuvante.

Además, sería importante aumentar la evaluación y publicaciones sobre la eficacia de los programas que ya se están llevando a cabo en diferentes instituciones para dar a conocer las ventajas de esta técnica en la práctica clínica.

\section{Referencias}

Alvariñas, M., \& Pino-Juste, M. (2019). Fases de un proyecto de investigación cualitativa: función de la triangulación. In A. P. Costa, M. C. Sánchez-Gómez \& M. V. M. Cilleros (Eds.), A práctica na investigaçao qualitativa: exemplos de estudos (pp. 23-42). Aveiro: Ludomedia.

Archambault, K., Vaugon, K., Deumie, V., Brault, M., Perez, R. M., Peyrin, J., Vaillancurt, G., \& Garel, P. (2019). MAP: a personalized receptive music therapy intervention to improve the affective well-being of youths hospitalized in a mental health unit. Journal of Music Therapy, 56(4), 381-402.

http://dx.doi.org/10.1093/jmt/thz013. 
Ashok, A., Shanmugam, S., \& Soman, A. (2019). Effect of music therapy on hospital induced anxiety and health related quality of life in coronary artery bypass graft patients: A randomised controlled trial. Journal of Clinical and Diagnostic Research, 13(11), YC05-YC09. https://doi.org//10.7860/JCDR/2019/42725.13274.

Barbeau, A. K., \& Cossette, I. (2019). The effects of participating in a community concert band on senior citizens' quality of life, mental and physical health. International Journal of Community Music, 12(2), 269-288. http://dx.doi.org/10.1386/ijcm.12.2.269_1.

Bittman, B., Poornima, I., Smith, M. A., \& Heidel, R. E. (2020). Gospel music: a catalyst for retention, engagement, and positive health outcomes for African Americans in a cardiovascular prevention and treatment program. Advances in Mind-Body Medicine, 34(1), 8-16.

Calo, F., Steiner, A., Millar, S., \& Teasdale, S. (2020). The impact of a community-based music intervention on the health and well-being of young people: a realist evaluation. Health \& Social Care in the Community, 28(3), 988-997. http://dx.doi.org/10.1111/hsc.12931.

Castro, E. M. C., Mota, M. P. G., Fonseca, S. C. F., Matos, A. P., \& Mourao-Carvalhal, M. I. (2020). Exercise with music: An innovative approach to increase cognition and reduce depression in institutionalized elderly. Revista de Psicologia del Deporte, 29(1), 49-56.

Chou, M., Chang, N., Chen, C., Lee, W., Hsin, Y., Siu, K., Chen, C. J., Wang, L. J., \& Hung, P. (2019). The effectiveness of music therapy for individuals with rett syndrome and their families. Journal of the Formosan Medical Association, 118(12), 1633-1643. http://dx.doi.org/10.1016/j.jfma.2019.01.001.

Clayton, K. D., Chumbler, N. R., Clark, C. N., Young, S. N., \& Willis, J. (2019). Patient-selected music rhythmically-paired with in-patient rehabilitation: a case report on an individual with acute stroke. Physiotherapy Theory and Practice, 37(2), 1-14. http://dx.doi.org/10.1080/09593985.2019.1628137.

Craig, P., Dieppe, P., Macintyre, S., Michie, S., Nazareth, I., \& Petticrew, M. (2008). Developing and evaluating complex interventions: the new Medical Research Council guidance. BMJ, 337(7676), 979983. http://dx.doi.org/10.1136/bmj.a1655.

Davies, C. R., Rosenberg, M., Knuiman, M., Ferguson, R., Pikora, T., \& Slatter, N. (2012). Defining arts engagement for population-based health research: art forms, activities and level of engagement. Arts \& Health, 4(3), 203-216. https://doi.org/10.1080/17533015.2012.656201.

Day, R. (2005). How to write \& publish a scientific paper. Washington: The Oryx Press.

Domínguez-Chávez, C. J., Murrock, C. J., Cavazos Guerrero, P. I., \& Salazar-Gonzalez, B. C. (2019). Music therapy intervention in community-dwelling older adults with mild cognitive impairment: A pilot study. Geriatric Nursing, 40(6), 614-619. http://dx.doi.org/10.1016/j.gerinurse.2019.06.004.

Ecer, H. D., \& Saritaş, S. (2019). The effects of music on the life signs of patients in the reanimation unit/recovery room after laparoscopic cholecystectomy. Holistic Nursing Practice, 33(5), 295-302. http://dx.doi.org/10.1097/HNP.0000000000000344.

Espí-López, G. V., Inglés, M., Ruescas-Nicolau, M., \& Moreno-Segura, N. (2016). Effect of low-impact aerobic exercise combined with music therapy on patients with fibromyalgia: a pilot study. Complementary Therapies in Medicine, 28, 1-7. http://dx.doi.org/10.1016/j.ctim.2016.07.003.

Fancourt, D. (2017). Arts in health: designing and researching interventions. Oxford: Oxford University Press.

Fancourt, D., \& Finn, S. (2019). What is the evidence on the role of the arts in improving health and well-being? A scoping review. Copenhagen: WHO Regional Office for Europe.

Ford, K., Tesch, L., Dawborn, J., \& Courtney-Pratt, H. (2018). Art, music, story: the evaluation of a person-centred arts in health programme in an acute care older persons' unit. International Journal of Older People Nursing, 13(2), e12186. http://dx.doi.org/10.1111/opn.12186.

Fujita, T., Ito, A., Kikuchi, N., Kakinuma, T., \& Sato, Y. (2016). Effects of compound music program on cognitive function and QOL in community-dwelling elderly. Journal of Physical Therapy Science, 28(11), 3209-3212.

Giuli, C., Paoloni, C., Santillo, E., Balietti, M., Fabbietti, P., Postacchini, D., \& Piacenza, F. (2020). Study of the effects of adapted tango and multidimensional intervention in prevention of dementia in aging: developing healthy lifestyle programs (STRENGTH project)-the experimental protocol of a prospective 
randomised controlled trial. Aging Clinical and Experimental Research, 32(12), 2329-2537.

http://dx.doi.org/10.1007/s40520-020-01504-4.

Gogoularadja, A., \& Bakshi, S. S. (2020). A Randomized study on the efficacy of music therapy on pain and anxiety in nasal septal surgery. International Archives of Otorhinolaryngology, 24(2), 232-236. http://dx.doi.org/10.1055/S-0039-3402438.

Gold, C., Eickholt, J., Assmus, J., Stige, B., Wake, J. D., Baker, F. A., \& Geretsegger, M. (2019). Music interventions for dementia and depression in Elderly care (MIDDEL): protocol and statistical analysis plan for a multinational cluster-randomised trial. BMJ Open, 9(3), 1-14. http://dx.doi.org/10.1136/bmjopen-2018-023436.

Gramaglia, C., Gambaro, E., Vecchi, C., Licandro, D., Raina, G., Pisani, C., \& Zeppegno, P. (2019). Outcomes of music therapy interventions in cancer patients: a review of the literature. Critical Reviews in Oncology/Hematology, 138, 241-254. http://dx.doi.org/10.1016/j.critrevonc.2019.04.004.

Gulliver, A., Pike, G., Banfield, M., Morse, A. R., Katruss, N., Pescud, M., \& West, S. (2019). Evaluation of the music engagement program for people with alzheimer's disease and dementia: study protocol for a pilot trial. Contemporary Clinical Trials Communications, 15, 1-10. http://dx.doi.org/10.1016/j.conctc.2019.100419.

Haase, U. (2012). Thoughts on WFMT's definition of music therapy. Nordic Journal of Music Therapy, 21(2), 194-195. http://dx.doi.org/10.1080/08098131.2012.678373.

Hall, T. L., Mullen, A., Plummer, J., Berry, S., \& Clancy, R. V. (2019). Sound practice: exploring the benefits of establishing a music group on an acute mental health inpatient unit. International Journal of Mental Health Nursing, 28(3), 697-705. http://dx.doi.org/10.1111/inm.12569.

Harvey, A. R. (2020). Links between the neurobiology of oxytocin and human musicality. Frontiers in Human Neuroscience, 14, 1-19. http://dx.doi.org/10.3389/fnhum.2020.00350.

Hernández, R. F. (2003). Metodología de la investigación. México: McGraw-Hill.

Hirschberg, R., Sylvia, L. G., Wright, E. C., Gupta, C. T., McCarthy, M. D., Harward, L. K., \& Spencer, T. J. (2020). Collaborative songwriting intervention for veterans with post-traumatic stress disorder. Journal of Alternative and Complementary Medicine, 26(3), 198-203. http://dx.doi.org/10.1089/acm.2019.0368.

Jauset-Berrocal, J. A. (2018). Música y neurociencia: la musicoterapia: fundamentos, efectos y aplicaciones terapéuticas. Espanha: Editorial UOC.

Johnson, J. K., Stewart, A. L., Acree, M., Napoles, A. M., Flatt, J. D., Max, W. B., \& Gregorich, S. E. (2020). A community choir intervention to promote well-being among diverse older adults: results from the community of voices trial. The Journals of Gerontology. Series B, Psychological Sciences and Social Sciences, 75(3), 549-559. http://dx.doi.org/10.1093/geronb/gby132.

Kayak, F., Unal, S., \& Yilmaz, E. (2016). Effects of relaxation exercises and music therapy on the psychological symptoms and depression levels of patients with schizophrenia. Archives of Psychiatric Nursing, 30(5), 508-512. http://dx.doi.org/10.1016/j.apnu.2016.05.003.

Kishida, M., Yamada, Y., Inayama, E., Kitamura, M., Nishino, T., Ota, K., \& Ikenoue, T. (2019). Effectiveness of music therapy for alleviating pain during haemodialysis access cannulation for patients undergoing haemodialysis: a multi-facility, single-blind, randomised controlled trial. Trials, 20(1), 1-12. http://dx.doi.org/10.1186/s13063-019-3773-x.

Kocaman, G., \& Benli, N. C. (2019). The effects of music therapy on vital signs and dental anxiety prior to dental surgery. Konuralp Tip Dergisi, 11(2), 308-313. http://dx.doi.org/10.18521/ktd.570696.

Koehler, F., Martin, Z., Hertrampf, R., Gaebel, C., Kessler, J., Ditzen, B., \& Warth, M. (2020). Music therapy in the psychosocial treatment of adult cancer patients: a systematic review and meta-analysis. Frontiers in Psychology, 11(651), 1-15. http://dx.doi.org/10.3389/fpsyg.2020.00651.

Koelsch, S. (2020). A coordinate-based meta-analysis of music-evoked emotions. NeuroImage, 223, 1-10. http://dx.doi.org/10.1016/j.neuroimage.2020.117350.

Kwok, S. Y. (2019). Integrating positive psychology and elements of music therapy to alleviate adolescent anxiety. Research on Social Work Practice, 29(6), 663-676. 
Lee, Y. J., Kim, M. A., \& Park, H. (2020). Effects of a laughter programme with entrainment music on stress, depression, and health-related quality of life among gynaecological cancer patients. Complementary Therapies in Clinical Practice, 39, 1-7. http://dx.doi.org/10.1016/j.ctcp.2020.101118.

Lin, C., Hwang, S., Jiang, P., \& Hsiung, N. (2020). Effect of music therapy on pain after orthopedic surgery-A systematic review and meta-analysis. Pain Practice, 20(4), 422-436. http://dx.doi.org/10.1111/papr.12864.

Loewy, J. (2020). Music therapy as a potential intervention for sleep improvement. Nature and Science of Sleep, 12, 1-9. http://dx.doi.org/10.2147/NSS.S194938.

Lopes-Junior, L. C., Bomfim, E. O., Nascimento, L. C., Nunes, M. D. R., Pereira-Da-Silva, G., \& Lima, R. A. G. (2016). Non-pharmacological interventions to manage fatigue and psychological stress in children and adolescents with cancer: an integrative review. European Journal of Cancer Care, 25(6), 921-935. http://dx.doi.org/10.1111/ecc.12381.

López, G., Christie, A. J., Powers-James, C., Bae, M. S., Dibaj, S. S., Gomez, T., \& Bruera, E. (2019). The effects of inpatient music therapy on self-reported symptoms at an academic cancer center: a preliminary report. Supportive Care in Cancer, 27(11), 4207-4212. http://dx.doi.org/10.1007/s00520-019-04713-4.

Low, M. Y., Lacson, C., Zhang, F., Kesslick, A., \& Bradt, J. (2020). Vocal music therapy for chronic pain: A mixed methods feasibility study. Journal of Alternative and Complementary Medicine, 26(2), 113-122. http://dx.doi.org/10.1089/acm.2019.0249.

MacRitchie, J., Breaden, M., Milne, A. J., \& McIntyre, S. (2020). Cognitive, motor and social factors of music instrument training programs for older adults' improved wellbeing. Frontiers in Psychology, 10, 119. http://dx.doi.org/10.3389/fpsyg.2019.02868.

Mandel, S. E., Davis, B. A., \& Secic, M. (2019). Patient satisfaction and benefits of music therapy services to manage stress and pain in the hospital emergency department. Journal of Music Therapy, 56(2), 149173. http://dx.doi.org/10.1093/jmt/thz001.

Millar, S. R., Steiner, A., Calo, F., \& Teasdale, S. (2020). COOL music: a 'bottom-up' music intervention for hard-to-reach young people in scotland. British Journal of Music Education, 37(1), 87-98. http://dx.doi.org/10.1017/S0265051719000226.

Miranda, M. C., Hazard, S. O., \& Miranda, P. V. (2017). La música como una herramienta terapéutica en medicina. Revista Chilena de Neuro-psiquiatría, 55(4), 266-277.

Moscoloni, N. (2005). Complementación metodológica para el análisis de datos cuantitativos y cualitativos en evaluación educativa. Revista Electrónica de Metodología Aplicada, 2(10), 1-10.

Packyanathan, J. S., Lakshmanan, R., \& Jayashri, P. (2019). Effect of music therapy on anxiety levels on patient undergoing dental extractions. Journal of Family Medicine and Primary Care, 8(12), 3854-3860. http://dx.doi.org/10.4103/jfmpc.jfmpc_789_19.

Page, M. J., McKenzie, J. E., Bossuyt, P. M., Boutron, I., Hoffmann, T. C., Mulrow, C. D., Shamseer, L., Tetzlaff, J. M., Akl, E. A., Brennan, S. E., Chou, R., Glanville, J., Grimshaw, J. M., Hróbjartsson, A., Lalu, M. M., Li, T., Loder, E. W., Mayo-Wilson, E., McDonald, S., McGuinness, L. A., \& Moher, D. (2021). The PRISMA 2020 statement: an updated guideline for reporting systematic reviews. BMJ, 372(71), 1-13. http://dx.doi.org/10.1136/bmj.n71.

Pedersen, M. R. V., Dam, C., \& Rafaelsen, S. R. (2020). Music and pain during endorectal ultrasonography examination: a prospective questionnaire study and literature review. Radiography, 26(3), 164-169. http://dx.doi.org/10.1016/j.radi.2020.01.004.

Penhune, V. B. (2020). A gene-maturation-environment model for understanding sensitive period effects in musical training. Current Opinion in Behavioral Sciences, 36, 13-22. http://dx.doi.org/10.1016/j.cobeha.2020.05.011.

Pérez, P. Z. (2011). Mixed method designs in education research: a particular experience. Revista Electrónica Educare, 15(1), 15-29. http://dx.doi.org/10.15359/ree.15-1.2.

Perkins, R., Ascenso, S., Atkins, L., Fancourt, D., \& Williamon, A. (2016). Making music for mental health: how group drumming mediates recovery. Psychology of Well-Being, 6(1), 1-17.

http://dx.doi.org/10.1186/s13612-016-0048-0. 
Philip, K., Lewis, A., \& Hopkinson, N. S. (2019). Music and dance in chronic lung disease. Breathe, 15(2), 116-120. http://dx.doi.org/10.1183/20734735.0007-2019.

Riggins, J., \& McLennon, S. M. (2020). Testing a musical game activity for community-dwelling older adults. Home Health Care Management \& Practice, 32(1), 22-27. http://dx.doi.org/10.1177/1084822319868703.

Rosado, A. (2019). Adolescents' experiences of music therapy in an inpatient crisis stabilization unit. Music Therapy Perspectives, 37(2), 133-140. http://dx.doi.org/10.1093/mtp/miz004.

Shankar, V. M., Geethanjali, B., Veezhinathan, M., Hariharakrishnan, J., Balakrishnan, N., \& Lakshmi, L. (2020). Evaluating the effect of music intervention on hypertension. Current Science, 118(4), 612-620. http://dx.doi.org/10.18520/cs/v118/i4/612-620.

Sharififard, N., Sargeran, K., Gholami, M., \& Zayeri, F. (2020). A music- and game-based oral health education for visually impaired school children; multilevel analysis of a cluster randomized controlled trial. BMC Oral Health, 20(1), 1-9. http://dx.doi.org/10.1186/s12903-020-01131-5.

Sheppard, A., \& Broughton, M. C. (2020). Promoting wellbeing and health through active participation in music and dance: a systematic review. International Journal of Qualitative Studies on Health and Wellbeing, 15(1), 1-15. http://dx.doi.org/10.1080/17482631.2020.1732526.

Skingley, A., McCue, J., \& Vella-Burrows, T. (2020). Using music interventions in the care of people with dementia. Nursing Standard, 35(6), 55-60. http://dx.doi.org/10.7748/ns.2020.e11560.

Spak, D., \& Card, E. (2019). Music, movement, and mind: use of drumming to improve strength, balance, proprioception, stamina, coordination, and emotional status in a 12-year-old with agenesis of the corpus callosum: a case study. Journal of Holistic Nursing, 38(2), 186-192. http://dx.doi.org/10.1177/0898010119871380.

Sylvia, Y., \& Kwok, L. (2018). Integratin positive psychology and elements of music therapy to alleviate adolescent anxiety. Research on Social Work Practice, 29 (6), 663-676.

Tian, Y., Ma, L., Xu, W., \& Chen, S. (2020). The influence of listening to music on adults with left-behind experience revealed by EEG-based connectivity. Scientific Reports, 10(1), 1-10. http://dx.doi.org/10.1038/s41598-020-64381-x.

Tillmann, A. C., Swarowsky, A., Correa, C. L., Andrade, A., Moratelli, J., Boing, L., \& Azevedo, G. A. C. (2020). Feasibility of a brazilian samba protocol for patients with parkinson's disease: a clinical nonrandomized study. Arquivos de Neuro-Psiquiatria, 78(1), 13-20. http://dx.doi.org/10.1590/0004$282 X 20190140$.

Travis Junior, R., Gann, E., Crooke, A. H. D., \& Jenkins, S. M. (2019). Hip hop, empowerment, and therapeutic beat-making: potential solutions for summer learning loss, depression, and anxiety in youth. Journal of Human Behavior in the Social Environment, 29(6), 744-765. http://dx.doi.org/10.1080/10911359.2019.1607646.

Uhlig, S., Jansen, E., \& Scherder, E. (2016). Study protocol RapMusicTherapy for emotion regulation in a school setting. Psychology of Music, 44(5), 1068-1081. http://dx.doi.org/10.1177/0305735615608696.

Valero-Cantero, I., Martinez-Valero, F. J., Espinar-Toledo, M., Casals, C., Barón-López, F. J., \& VázquezSánchez, M. Á. (2020). Complementary music therapy for cancer patients in at-home palliative care and their caregivers: protocol for a multicentre randomised controlled trial. BMC Palliative Care, 19(1), 61. https://doi.org/10.1186/s12904-020-00570-9.

Vinciguerra, C., De Stefano, N., \& Federico, A. (2019). Exploring the role of music therapy in multiple sclerosis: brief updates from research to clinical practice. Neurological Sciences, 40(11), 2277-2285. http://dx.doi.org/10.1007/s10072-019-04007-x.

Wang, Y., Zheng, T., Liao, Y., Li, L., \& Zhang, Y. (2020). A meta-analysis of the effect of music therapy on Alzheimer's disease. International Journal of Clinical and Experimental Medicine, 13(2), 317-329.

Wei, T., Tian, X., Zhang, F., Qiang, W., \& Bai, A. (2020). Music interventions for chemotherapy-induced nausea and vomiting: a systematic review and meta-analysis. Supportive Care in Cancer, 28, 4031-4041. http://dx.doi.org/10.1007/s00520-020-05409-w. 
Windle, E., Hickling, L. M., Jayacodi, S., \& Carr, C. (2020). The experiences of patients in the synchrony group music therapy trial for long-term depression. The Arts in Psychotherapy, 67, 1-44. http://dx.doi.org/10.1016/j.aip.2019.101580.

World Health Organization - WHO. (2018). Non communicable diseases. Recuperado el 3 de marzo de 2021, de https://www.who.int/news-room/fact-sheets/detail/noncommunicable-diseases

Ye, W. (2020). Regulating effect of musical education on the positive emotions considering the effect of emotional contagion. Revista Argentina de Clínica Psicológica, 29(1), 322-327. http://dx.doi.org/10.24205/03276716.2020.43.

Zimpel, S. A., Torloni, M. R., Porfírio, G. J. M., Flumignan, R. L. G., \& Silva, E. M. K. (2020). Complementary and alternative therapies for post-caesarean pain. Cochrane Database of Systematic Reviews, 2020(9), 1-147. http://dx.doi.org/10.1002/14651858.CD011216.pub2.

\section{Contribución de los Autores}

Las tres autoras trabajaron de forma síncrona realizando la búsqueda en las diferentes plataformas, seleccionando los artículos objeto de estudio y diseńando una base de datos en Excel. Sara Domínguez-Lloria analizó los datos cuantitativos y redactó el primer informe de resultados, María José González-Ojea redactó la discusión y Margarita Pino-Juste redactó la versión final del documento. Todos los autores aprueban la version final del texto.

\section{Autor para la correspondencia}

Sara Domínguez-Lloria

e-mail: saradominguez.lloria@usc.es

\section{Editor de sección}

Prof. Dr. Rodolfo Morrison 